\title{
On the Assimilation of Cloud Condensational Heating in Prediction Models
}

\author{
By Huw C. Davies \\ Atmospheric Physics, ETH Zürich, Switzerland \\ (Manuscript received 4 November 1986; in revised form 12 January 1987)
}

\begin{abstract}
Some issues that arise in relation to the assimilation of cloud condensational heating in mesoscale prediction models are considered from a theoretical standpoint. A study is undertaken of the response of a simple atmospheric flow model to various forms of externally prescribed, mesoscale, steady, zero horizontally or area-averaged diabatic heating distributions. It is shown that the amplitude and/or phase of the response exhibits strong sensitivity to the depth of the "cloud" layer and to the shape of the vertical diabatic profile within the layer. On the premise that sustenance of the cloud system requires a phase match between the low-level convergence and the diabatic heating rate a search is made for forms of vertical diabatic profiles that satisfy this criterion. The results gleaned from this inverse approach are consistent with contentions that the contribution of evaporative cooling in a sub-cloud layer plays an important role in determining the response of "shallow" systems, and that the relative disposition of condensational heating between the upper and lower regions of the cloud layer is an important factor for "deep" systems. The general applicability of these inferences is limited by the idealised nature of the theoretical model employed in the study. At the very least, they serve as tentative, cautionary remarks on the issue of the assimilation of condensational heating effects in NWP models.
\end{abstract}

\section{Introduction}

A range of mesoscale observational data sets are now routinely acquired in quasi-real time (see e.g. Tatehira et al., 1981; Roesli, 1981), and are in principle available as input data for mesoscale NWP models. These data sets are most likely to comprise a combination of, or all of the following: satellite derived visible and IR cloud images, reflectivity patterns from a network of conventional weather radars, and standard data from a network of mesoscale-spaced automatic surface stations. All three of these sources provide information, albeit indirect, on the condensational heating experienced by the atmosphere. It is therefore natural to ponder what utility lies in estimating this diabatic heating distribution from the fore- going space-time observational data and feeding this information to an NWP mesoscale model run in a "data-assimilation" mode.

The outcome of such an undertaking will depend upon several interrelated technical, numerical modelling and physical factors. In particular it will be related to the quantitative accuracy of the derived diabatic estimates, the efficiency of the assimilation scheme used in the forecast model and the subtlety (and magnitude) of the dynamical interaction between the flow and the diabatic heating.

The core of the present study is an exploration of one aspect of this dynamical interaction. We consider the nature of the flow response to mesoscale condensational heating distributions, and examine the possible infiuence of errors in the specification 
of the heating upon the response. The approach is conceptionally simple and heuristic: -adopt a simple theoretical flow model, prescribe externally various forms of space-time diabatic distributions, examine the sensitivity and physical plausibility of the flow response. This strategy will shed some light upon, and enable us to draw some cautionary inferences related to the nature of the feedback. However the highly idealised model used in the study severely curtails the validity/generality of these inferences.

This theoretical approach is somewhat in contrast to, but complements and is prompted by, the usual "Nowcasting" and "Numerical Model" approaches to issues of mesoscale forecasting. The backbone of the former is the semi-empirical estimate of the surface rainfall rate from rader and satellite information. (Careful assessments of the limitations, current status and some possible future developments in this field can be gleaned from the studies of Zawadzki (1984), Austin (1985) and Tsonis and Isaac (1985)). For our present purpose it is of interest to note for instance that rainfall estimates based only on radar measurements have an expected error on the order of $30 \%$.

In the second, NWP-type approach there are practical reasons for desiring to explicitly account for condensational heating effects in the initial state of a mesoscale NWP model. These stem from the recognition that the initialization procedure for a short range forecast should serve to ensure that the forecast is not hampered by spurious initial imbalances and moreover produces a reasonable forecast product even in the first few hours of model simulation. Some studies related to this objective have already been undertaken. Tarbell et al. (1981), using a static initialization scheme for a mid-latitude forecast model, included a diabatic term in their formulation of the omega equation. Fiorino and Warner (1981) and Molinari (1982) with hurricane models, and Danard (1985) with a mid-latitude model, demonstrated the positive impact upon the forecast of an assimilation period carried out with an externally specified diabatic heating distribution. More recently
Ninomiya (1987) and Ueno et al. (1987) report similar experiments with a short $(\sim 1 \mathrm{hr})$ assimilation period. Their results show a major alleviation of the model spin-up problem with a concomitant improvement in the short term precipitation forecast. Some related work for the tropics based upon satellite imagery is also currently underway (Kasahara, private communication). In all these model experiments the net condensational heating along with the horizontal distribution and depth of the active cloud is estimated from observations, whereas the vertical profile of the heating is choosen with one of a variety of pragmatic techniques. It is of particular interest to note that according to Ninomiya the flow development is sensitive to the form of this vertical profile.

The spur for this present study derives from these two forementioned strands of unavoidable errors in the estimates of the net heating, and the accompanying uncertainty in the specification of the spatial dimension and vertical profile of the heating. The present study constitutes a preliminary and rudimentary theoretical examination of the possible impact of these uncertainties.

\section{Diagnosis of the diabatic flow response}

\subsection{Details of the Model}

The model system represents incompressible, inviscid and stratified flow above an $f$ plane. The equations are linearised for two dimensional hydrostatic perturbations about a basic state of uniform flow $(U)$ and uniform state stability (i.e. the equivalent of a constant Brunt-Vaisala frequency, $N$ ). It follows that the governing equations for the perturbation variables of our system take the following form,

$$
\begin{array}{cl}
u_{t}+U u_{x}-f v=-p_{x}^{*} & \text { (a) } \\
v_{t}+U v_{x}+f u=0 & \text { (b) } \\
p_{z}^{*}=\theta^{*} & \text { (c) } \\
u_{x}+w_{z}=0 & \text { (d) } \\
\theta_{t}^{*}+u \theta_{x}^{*}+N^{2} w=N^{2} h & \text { (e) }
\end{array}
$$

where $(u, w)$ denote the perturbation horizontal and vertical velocities, and $\left(\theta^{*}, p^{*}\right)$ 
$\left\{(g \theta / \Theta),\left(p / \rho_{0}\right)\right\}$ are respectively scaled measures of potential temperature and pressure. Here $\Theta$ and $\rho_{0}$ refer to the base values of potential temperature and density, and finally $N^{2} h=\left(g Q / c_{p} T_{0}\right)$ is the diabatic contribution linked with $a$ heating rate $Q$ and base temperature $T_{0}$.

The strategy adopted here is to specify the diabatic distribution $Q$ as an external parameter and then diagnose the response of the model atmosphere. For simplicity we assume that $Q$ has a wavelike form in the horizontal with a zero horizontal mean value (i.e. diabatic heating and cooling). Thus a single wave component of the diabatic effect can be written as

$$
h=\eta(z) e^{i(k x-\omega t)}
$$

i.e. a propagating wave with a specified frequency $(\omega)$, horizontal wavenumber $(k)$ and vertical structure $\eta(z)$. Our objective is to determine the sensitivity of the response to variations in the specification of these external parameters.

In view of (2.2) the system $(2.1 \mathrm{a}-\mathrm{e})$ can be combined to form a single equation:

$$
W_{z z}+\lambda^{2} W=\lambda^{2} \eta
$$

where

$$
\begin{aligned}
& w=W(z) e^{i(k x-\omega t)}, \\
& \lambda^{2}=N^{2} k^{2} /\left(\tilde{\omega}^{2}-f^{2}\right), \\
& \widetilde{\omega}=(\omega-U k) .
\end{aligned}
$$

Here $W$ is the amplitude of the vertical velocity response, $\lambda$ is a measure of the vertical wavenumber, and $\tilde{\omega}$ is the doppler-shifted frequency. Note that, for a Rossby number defined by $R_{0}=|\widetilde{\omega} / f|$, then $\lambda^{2} \lessgtr 0$ for $R_{0} \lessgtr 1$. The upper and lower inequalities correspond respectively to evanescent and wave-like vertical structures in the $\eta \equiv 0$ flow domains.

To cast light on the nature of the flow response to the form of $\eta(z)$ we examine in the next three sub-sections various classes of vertical profiles.

\subsection{Symmetric Diabatic Profiles}

Here we consider a set of four profiles for $\eta(z)$ for each of which

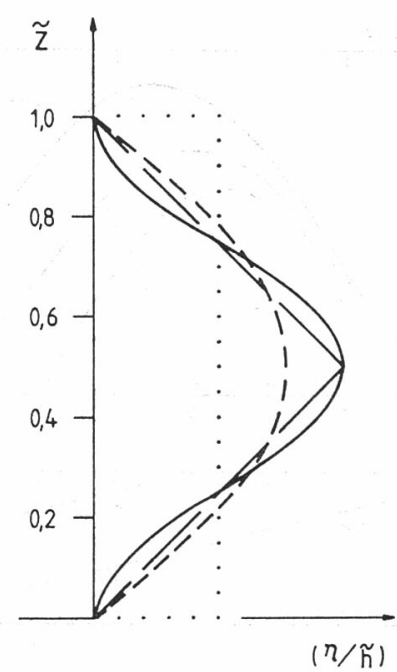

Fig. 1 The normalized vertical structure of diabatic heating corresponding to the profiles (a), (b), (c) and (d) of Eqs. 2.5. The profiles are represented respectively by light solid, long dashed, short dashed and dotted lines.

-the diabatic heating is non-zero only in a limited vertical domain $(z \leqq d)$ adjacent to the ground, i.e. $\eta(z) \equiv 0$ for $(z / d)>1$, and

- the profile within this layer is symmetric about mid-cloud level, $z=(1 / 2) d$.

The selected profiles are shown in Fig. 1 and have the following forms,
(a) $\eta(\tilde{z})=\tilde{h}[1-\cos (2 \pi \tilde{z})]$ for $\tilde{z} \leqq 1$,
(b) $\eta(\tilde{z})=4 \tilde{h} \tilde{z}$ for $0 \leqq \tilde{z} \leqq 1 / 2$, $=4 \tilde{h}(1-\tilde{z})$ $1 / 2 \leqq \tilde{z} \leqq 1$,

(c) $\eta(\tilde{z})=(1 / 2) \pi \tilde{h} \sin (\pi \tilde{z})$ for $\tilde{z} \leqq 1$,

(d) $\eta(\tilde{z})=\tilde{h} \quad$ for $\tilde{z} \leqq 1$,

where

$\tilde{z}=z / d, \quad$ and $\tilde{h}$ is a constant.

The profiles have been normalised to ensure the same net total diabatic amount in a vertical column.

Equation (2.3) is to be solved for each of these profiles subject to a zero vertical velocity condition at $z=0$ and a radiation condition off at infinity. Solutions are derived separately for $\tilde{z} \gtrless 1$ and matched by applying the appropriate boundary conditions at the 

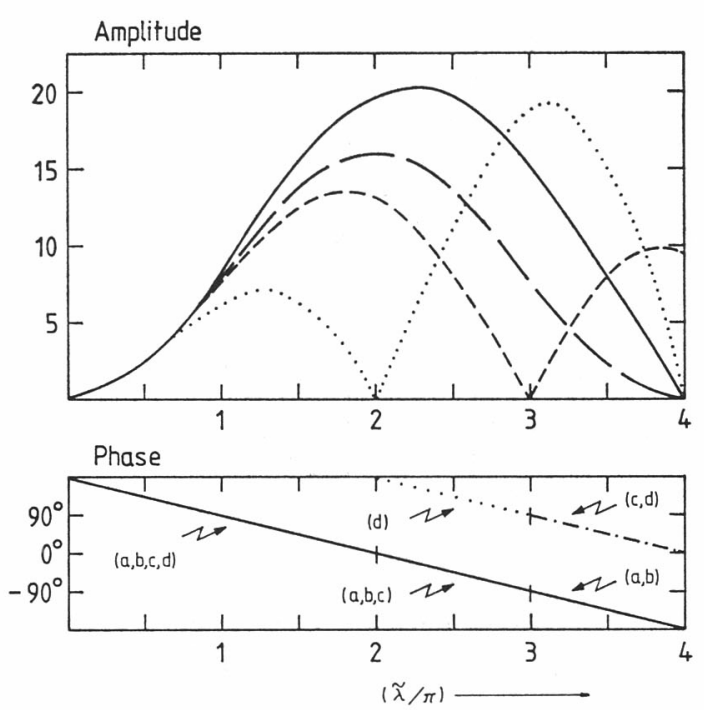

Fig. 2 The amplitude (upper diagram) and phase (lower diagram) of the horizontal convergence at the ground displayed as a function of $(\tilde{\lambda} / \pi)$ for diabatic forcing corresponding to the four profiles of Fig. 1 (the line coding is as in that figure). The parameter $\tilde{\lambda}$ is the product of the cloud depth and the natural vertical wavelength assocaited with an $R_{0}>1$ regime. (Note that for quasi-steady mesoscale diabatic forcing then $(\lambda / \pi)=1,2,3, \cdots \cdots$ correspond to cloud depths of $3,6,9, \cdots \mathrm{km}$ ).

layer interface. (An additional matching at $\tilde{z}=1 / 2$ is required for profile b).

In terms of the possible feedback and maintenance of the condensational heating one revealing measure of the flow response is the value of the horizontal convergence $\left(w_{\bar{z}}\right)$ at the ground. The amplitude and phase of this flow variable for the various profiles is shown in Fig. 2, and correspond to the following formulae:

(a) $\left.\left(w_{\tilde{z}} / \tilde{h}\right)\right|_{\tilde{z}=0}$ $=-2 \tilde{\lambda}\left[1-(\tilde{\lambda} / 2 \pi)^{2}\right]^{-1} \sin \{(1 / 2) \tilde{\lambda}\} e^{-i(1 / 2) \tilde{\lambda}}$,

(b) $\left.\left(w_{\tilde{z}} / \tilde{h}\right)\right|_{\tilde{z}=0}=-16 \sin ^{2}\{(1 / 4) \tilde{\lambda}\} e^{-i(1 / 2) \tilde{\lambda}}$,

(c) $\left.\left(w_{\tilde{z}} / \tilde{h}\right)\right|_{\tilde{z}=0}$

$$
=-\tilde{\lambda}^{2}\left[1-(\tilde{\lambda} / \pi)^{2}\right]^{-1} \cos \{(1 / 2) \tilde{\lambda}\} e^{-i(1 / 2) \tilde{\lambda}},
$$

(d) $\left.\left(w_{\bar{z}} / \tilde{h}\right)\right|_{\tilde{z}=0}=-2 \tilde{\lambda} \sin \{(1 / 2) \tilde{\lambda}\} e^{-i(1 / 2) \tilde{\lambda}}$,

where $\tilde{\lambda}=(\lambda d)$ is the ratio of the cloud depth to $(1 / 2 \pi) \times$ (the natural vertical wavelength of the system). Hereafter we shall refer somewhat loosely to $\tilde{\lambda} \gtrless 1$ as "deep" and "shallow" diabatic distributions. In interpreting these results it might also be helpful to note that for stationary, meso- $\beta$ scale diabatic forcing (i.e. $\omega \rightarrow 0, R_{0}=|U k / f| \gg 1$ ) the vertical wavenumber can be approximated by the relation $\lambda=N / U$. Then for typical values of $N$ and $U$ the parameter values $(\tilde{\lambda} / \pi)=1,2,3 \cdots$ etc. correspond to cloud depths of $3,6,9 \mathrm{~km} \cdots$, . This particular parameter limit $\left(\omega \rightarrow 0, R_{0} \gg 1\right)$ is also significant from another standpoint. The implied horizontal scape independence of $\tilde{\lambda}$ indicates that we can consider at least in qualitative terms not only single wave components of the form (2.2) but also meso- $\beta$ scale wave packets, i.e. strong localised diabatic heating and weak widespread cooling. In this subsection we confine ourselves to the $R_{0}>1$ regime and hence to $\tilde{\lambda}^{2}>0$.

It is evident from Fig. 2 that, for $\tilde{\lambda} \leqslant \pi$, all four profiles produce qualitatively similar responses. The low-level convergence and the accompanying vertical velocity field is out of phase with the diabatic heating rate i.e. ascent (descent) in regions of cooling (heating), whilst the amplitude is proportional to $\tilde{\lambda}^{2}$. This uniformity of response breaks down as $\tilde{\lambda}$ increases and substantial departures are evident respectively for profiles (d) and (c) in the neighbourhood of $(\tilde{\lambda} / \pi) \sim 2$ and 3. Note also the in-phase response of all the profiles at $(\tilde{\lambda} / \pi)=2$.

These results bear upon and relate to several previous studies. A phase mis-match between vertical velocity and heating in the $\omega=0$, meso- $\beta$ scale limit was noted by Smith and Lin (1982), whilst the in-phase response at $(\tilde{\lambda} / \pi)=2$ is consistent with the wave-CISK criterion of Chang (1976) and Davies (1979, 1980). In a CISK context, the zero-amplitude of profile (d) at precisely $(\tilde{\lambda} / \pi)=2$ sets apart this profile and suggests that its adoption in theoretical wave-CISK studies might not be appropriate. Two studies, those of Hayashi (1976) and Raymond (1986), relate respectively to our consideration of profiles (c) and (d). In the former study Hayashi noted a large amplitude response at (in our notation) $\tilde{z}=3 / 4$ for $(\tilde{\lambda} / \pi)=1$. This enhanced "upper-cloud 
level" response, although present, is not evident in the surface pattern but is symptomatic of a rich vertical structure. In the other study, Raymond considered the response to local diabatic effects in both steady state and transient configurations, and his results for the steady state problem also parallel ours. However, he takes the absence of strong in-phase response for his particular profile (profile d) as a general indicator of the inefficiency of conventional wave-CISK. This interpretation should be viewed with some reserve in the light of the foregoing cautionary remarks regarding profile (d).

In relation to our present study the results displayed in Fig. 2 are disconcerting on two accounts. Firstly the large out-of-phase behaviour for an external cloud specification that corresponds to a $(\tilde{\lambda} / \pi) \ll 2$ regime is not compatible with condensational heating sustenance via low-level moisture convergence. Secondly the results for $(\tilde{\lambda} / \pi) \gtrsim 3$ betoken a strong sensitivity to the vertical profile of the diabatic heating even when the anomalous profile (d) is excluded. However, the response for the profiles (a) and (b) do show a not unreasonable qualitative correspondence across the entire displayed span of $\tilde{\lambda}$. It is in the light of this latter harmony that we select a variant of one of these profiles (profile b) to examine the effects of differing ratios of condensational heating distribution between the upper and lower regions of the diabatic layer.

\subsection{An Asymmetric Diabatic Profile}

Consider the following asymmetric extension of profile (b)

$$
\text { (e) } \begin{array}{rlrl}
\eta(\tilde{z}) & =2(\tilde{h} / p) \tilde{z} & \text { for } & 0<\tilde{z} \leqq p \\
& =2[\tilde{h} /(1-p)](1-\tilde{z}) & p \leqq \tilde{z} \leqq 1 \\
& =0 & \tilde{z}>1 .
\end{array}
$$

i.e. a picewise linear diabatic profile as before, but now with the level of maximum heating amplitude located at an arbitrary dimensionless height, $\tilde{z}=p$, within the cloud.

To assess the response we consider as before the low level horizontal convergence $\left(w_{z}\right)$ at the ground, and also the vertical velocity at the level of maximum heating. The respective formulae are given by,

$$
\begin{aligned}
& \left.\left(w_{\tilde{z}} / \tilde{h}\right)\right|_{\tilde{z}=0} \\
& =(2 / p)\left\{1+(1-p)^{-1}\left(p e^{-i \tilde{\lambda}}-e^{-i p \tilde{\lambda}}\right)\right\},
\end{aligned}
$$

and

$$
\begin{aligned}
& \left.(w / \tilde{h})\right|_{\tilde{z}=p} \\
& =(2 / p)\left\{p+(1-p)^{-1} \tilde{\lambda}^{-1}\right. \\
& \left.\sin (p \tilde{\lambda})\left(p e^{-i \tilde{\lambda}}-e^{-i p \tilde{\lambda}}\right)\right\} .
\end{aligned}
$$

First consider the response in the high Rossby number $\left(R_{0}>1\right)$ regime. Fig. 3 shows the amplitude and phase of the low-level convergence in the parameter space of $(\tilde{\lambda}, p)$ for $(\tilde{\lambda} / \pi)=(0-4)$ and $p=(0.1-0.9)$, and Fig. 4 is a similar diagram for the vertical velocity, $\left.(w / \tilde{h})\right|_{\tilde{z}=p}$. At small values of $(\tilde{\lambda} / \pi)$ the amplitude of the response at both levels is

Horizontal convergence at ground level
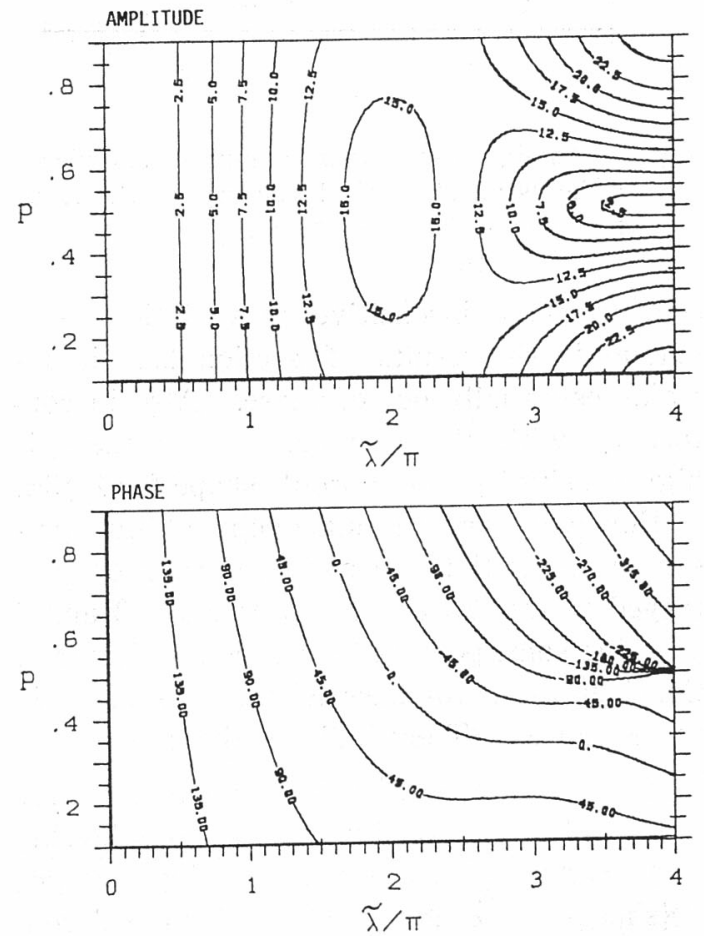

Fig. 3 Amplitude and phase of the horizontal convergence at ground level displayed in the parameter space of $(\tilde{\lambda}, p)$ for an $R_{0}>1$ regime. The diabatic vertical profile corresponds to that given by Eq. 2.7. Here $\tilde{z}=p$ refers to the dimensionless height (i.e. scaled by cloud depth) of the level of maximum amplitude of diabatic heating. 
Vertical velocity at level of maximum heating AMPLITUDE

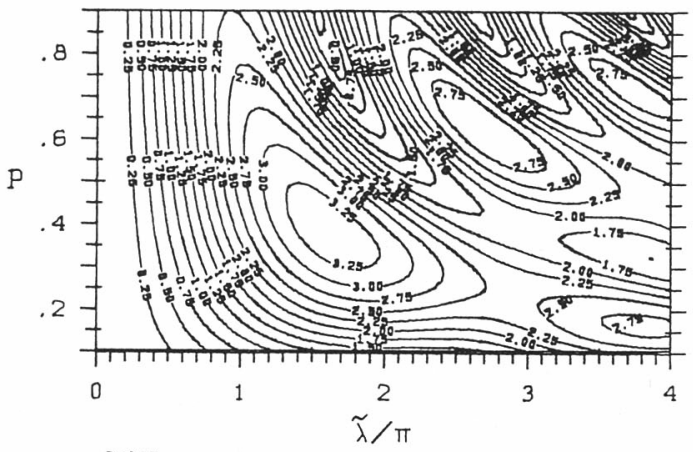

PHASE

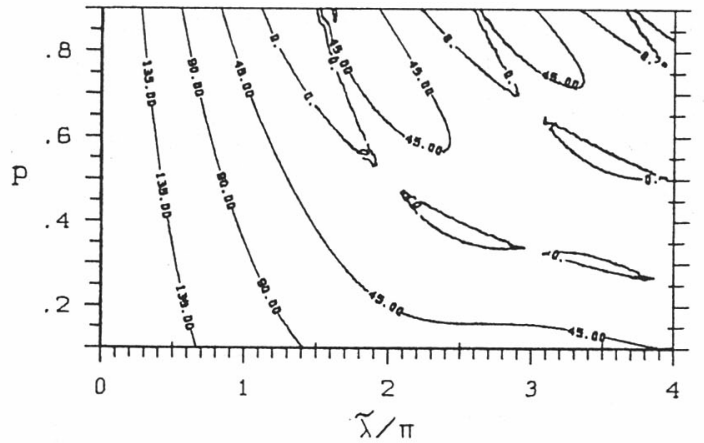

Fig. 4 As Fig. 3 but for the amplitude and phase of the "scaled" vertical velocity at the level of maximum heating $(z=p)$.

comparatively insensitive to variations in $p$ but, as in the results of section 2.2 , it remains essentially out of phase. Also in conformity with the previous results there is high sensitivity to the profile shape for $\tilde{\lambda} \geq 3 \pi$.

However from the standpoint of our present investigation the phase information portrayed in the lower panels of Figs. 3 and 4 contains some potentially redeeming features. Consider the zero-phase isolines displayed in these figures. They indicate that:

-at the surface (Fig. 3) there is now, for any value of $\tilde{\lambda} \geqq(3 / 2) \pi$, one (or more) level(s) of maximum heating $(\tilde{z}=p)$ given by $p=$ $\{\sin (p \tilde{\lambda}) / \sin \tilde{\lambda}\}$ for which the low-level convergence is exactly in-phase with the diabatic heating, and

-at the level of maximum heating (Fig. 4) there are two such families of zero-phase isolines. In this case these isolines signify an exact phase-match between the vertical velocity at this level and the diabatic heat- ing. The two families of isolines are given be

$$
\begin{aligned}
& \quad p=\sin (p \tilde{\lambda}) / \sin \tilde{\lambda}, \\
& \text { and } \\
& \quad p=(2 n-1)(\tilde{\lambda} / \pi)^{-1} \text { with } n=1,2, \cdots .
\end{aligned}
$$

Note that there is a narrow band in $(\tilde{\lambda}, p)$ space adjacent to these neighbouring isoline families for which there is only a small phase difference between $\left.w\right|_{\bar{i}=p}$ and the diabatic heating. For the first family of isolines there is an exact vertical phase coherence between the vertical velocity at low-level and that at the level of maximum heating, whilst for the second family (with $n=1$ ) the vertical wavelength, $(2 \pi / \tilde{\lambda})$, is twice the height of the level of maximum heating. Also in this latter case $\left.(w / \tilde{h})\right|_{i=p}=2.0$. This signifies in cloud parameterization terminology a cloud mass flux at the level of maximum heating that is twice that of the mesoscale response of the vertical velocity field at the same level.

Thus, in terms of the issue of the assimilation of condensational heating effects, the sensitivity of the amplitude response to the shape of $\eta(\tilde{z})$ for $(\tilde{\lambda} / \pi)>3 / 2$ is countered by the existence of particular profile shapes, with given $p$ values, that can sustain a ' $w-h$ ' in-phase response at low-level and an accompanying vertical phase coherency of the ascending air. This result is of some interest if it is argued that the foregoing phase relationships are desirable on physical grounds and should be sought for, or enforced, in an NWP assimilation run incorporating condensational heating.

Now consider the nature of the response of the idealised atmosphere for the low Rossby number regime $\left(R_{0}<1\right)$. It is probably the response to forcing in this regime that is predominantly encountered in most current NWP models.

Fig. 5 displays the response of both the low-level convergence and of the vertical velocity at $\tilde{z}=p$. Now the results are portrayed in the parameter space of $(\tilde{\mu}, p)$, where $\tilde{\mu}^{2}=-\tilde{\lambda}^{2}$. Note that for $\left\{R_{0}<1\right\}$ then $\mu \approx(N k / f)$ and hence values of $(\tilde{\mu} / \pi)=1,2,3$, approxi- 
/W/ at level of maximum heating

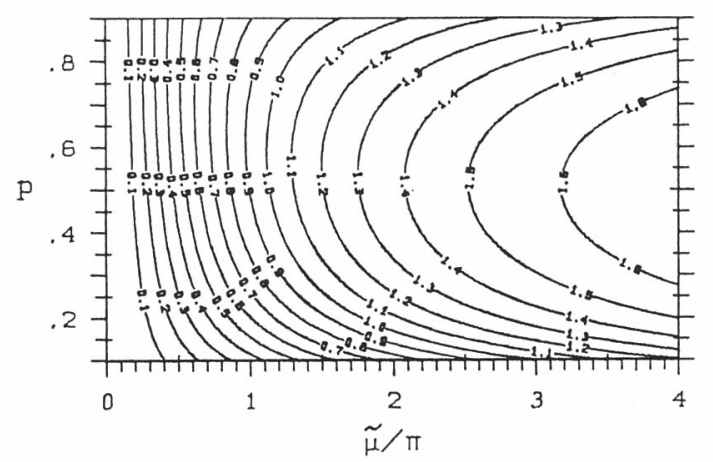

$/ W_{z} /$ at ground level

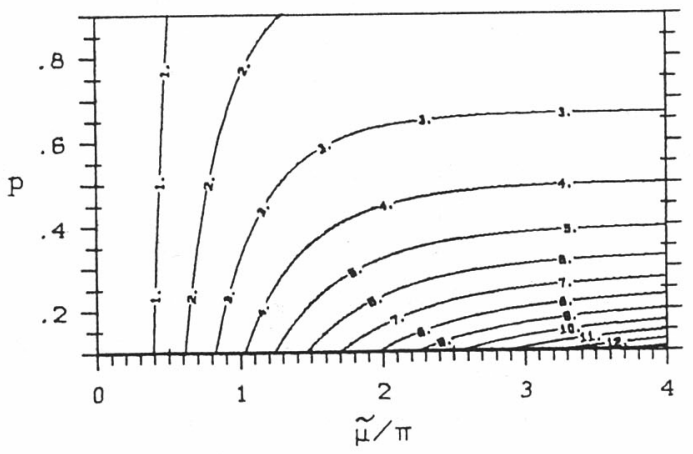

Fig. 5 The scaled amplitude response of the vertical velocity at the level of maximum heating (upper diagram) and the ground level convergence (lower diagram) displayed in the parameter space of $(\tilde{\mu}, p)$. Here $\tilde{\mu}$ is the product of the cloud depth with the natural vertical exponential decay rate associated with an $R_{0}<1$ regime. (See text for an indication of the values of the cloud aspect ratio associated with $\tilde{\mu}$ ).

mate respectively to heating distributions with "cloud depth-to-horibontal wavelength" ratios of $(0.5,1.0,1.5) .10^{-3}$. There is now no phase shift with height so that $w$ and $h$ are in phase at all cloud levels. In broad terms the results indicate that it is variations in $\tilde{\mu}$ (i.e. essentially in the specification of the aspect ratio) as opposed to $p$, that are more sensitive for $\tilde{\mu} \ll \pi$. The reverse holds true at the ground for $\tilde{\mu} \gg \pi$. This degree of sensitivity is not insignificant e.g. for $p \leqq 0.4$ a fractional error of $20 \%$ in the specification of "cloud" depth for an aspect ratio $\sim(0.5)$ $10^{-2}$ modifies the response by a factor of $\sim 2$, whilst a $20 \%$ variation in $p$ at aspect ratios (1.5) $10^{-2}$ will induce $\sim 20 \%$ change in the response. These amplitude variations are such as to merit further attention, but one should also bear in mind the crudity of the the model (e.g. Ekman p.b.l. effects are likely to be strong and to increase as $\tilde{\mu}$ decreases).

\subsection{Another Enriched Vertical Profile}

In this section we extend our study of the $(\tilde{\lambda} / \pi) \ll 1$ regime. Our motivation is to examine in the context of the present model the influence of sub-cloud layer condensational cooling upon the previously noted low-level ' $w-h$ ' phase mis-match that occurs in this regime. To this end consider the following "double sinusoidal segment" profile for the diabatic term,

$$
\text { (f) } \begin{array}{rlrl}
\eta(\tilde{z}) & =-\frac{1}{2} \pi \tilde{h}(\delta / m) \sin (\pi \tilde{z} / m) \\
& \text { for } 0<\tilde{z}<m, \\
& =\frac{1}{2} \pi \tilde{h} \sin \{\pi(\tilde{z}-m)\} \\
& \text { for } & m \leqq \tilde{z} \leqq(1+m), \\
= & \tilde{z} \geqq(1+m),
\end{array}
$$

Now our usual heating region is elevated to the layer $[m, 1+m]$ and it surmounts a cooling layer of height ' $m$ '. This is a crude representation of sub-cloud layer condensational cooling in evaporating downdraughts. (Note however that an unphysical "sub-cloud" layer warming is also implied by 2.2 and 2.10). The parameter ' $\delta$ ' indicates the fraction of the net latent heat released in the upper layer that is reabsorbed in the subcloud layer (i.e. $\delta \leqq 1$ ).

For this profile it is appropriate to examine the vertical velocity at "cloud" base $\tilde{z}=m$. This variable satisfies the relationship

$$
\begin{aligned}
& \left.(w / \tilde{h})\right|_{\tilde{z}=m} \\
& =\frac{1}{2} \tilde{\lambda} \sin (m \tilde{\lambda})\left\{\delta\left[1-(m \tilde{\lambda} / \pi)^{2}\right]^{-1}\right. \\
& \left.\quad-\left[1-(\tilde{\lambda} / \pi)^{2}\right]^{-1}\left(1+e^{-i \tilde{\lambda}}\right)\right\} e^{-i m \tilde{\lambda}}
\end{aligned}
$$

Here we shall not present a comprehensive analysis of this equation but merely consider some special cases. First note that if $\delta \equiv 0$ this expression can be reduced to 


$$
\begin{aligned}
\left.(w / \tilde{h})\right|_{\tilde{z}=m} & =-\tilde{\lambda}\left[1-(\tilde{\lambda} / \pi)^{2}\right]^{-1} \\
& \cdot \sin (m \tilde{\lambda}) \cos \left(\frac{1}{2} \tilde{\lambda}\right) e^{-i(1 / 2+m) \tilde{\lambda}},
\end{aligned}
$$

and comparison of (2.12) with (2.6c) indicates that, for $(m \tilde{\lambda} / \pi) \leqslant 1 / 2$, the response differs little from that for profile (c). This result provides, at least for the case of zero evaporation beneath cloud base, some justification for our previous omission of a sub-cloud layer.

Another special case of some interest is that of equal depth to the cloud and subcloud layers (i.e. $m=1$ ). In this case

$$
\begin{aligned}
&\left.\left(w_{\dot{z}} / \tilde{h}\right)\right|_{\tilde{z}=0}=-\frac{1}{2} \tilde{\lambda}^{2}\left[1-(\tilde{\lambda} / \pi)^{2}\right]^{-1} \\
& \cdot\left\{\delta-2 \cos \left(\frac{1}{2} \tilde{\lambda}\right) e^{-(1 / 2) \lambda}\right\} e^{-i \lambda},
\end{aligned}
$$

and hence the in-phase response that prevailed previously for $\tilde{\lambda}=2 \pi$, is not necessarily eliminated by the evaporational cooling in the "sub-cloud" layer. Of more immediate interest for our present objective is the deduction from (2.11) of the existence of in-phase response for $(m \tilde{\lambda})<\pi$, when the following two criteria are met

$$
\begin{aligned}
\delta\left[1-(m \tilde{\lambda} / \pi)^{2}\right]^{-1} & =\left[1-(\lambda / \pi)^{2}\right]^{-1}, \\
\text { and } \quad(m+1) \tilde{\lambda} / \pi & =1 .
\end{aligned}
$$

The first condition requires that $(m>1)$, i.e. depth of sub-cloud layer exceeds that of the cloud layer. This result is one example indicating that an in-phase response between the vertical velocity at cloud base and the diabatic heating can be attained, even for shallow cloud depths. For this case the precipitation efficiency must assume the particular value implied by (2.14) viz

$$
\delta=\lambda^{*}\left(2-\lambda^{*}\right)\left(1-\lambda^{* 2}\right),
$$

where $\lambda^{*}=(\tilde{\lambda} / \pi)$. However there may be other examples of such an in-phase response in the parameter space of $\{\tilde{\lambda}, m, d\}$.

In the light of these results and the ones derived in the previous section it is tempting to suggest that "sub-cloud" layer evaporative cooling might play a significant role in mesoscale, shallow $(\tilde{\lambda} \leq 1)$, steady-state convective systems. Certainly the derived results indi- cate that the existence of such cooling might induce a radically different mode of response.

\section{Further remarks}

In the previous section a study was made of the response of a simple atmospheric flow model to various forms of externally, prescribed, mesoscale, steady, and zero areaaveraged' diabatic distributions. The results obtained with vertically symmetric "in-cloud" diabatic profiles show that for $R_{0}>1$ there is on the one hand a large phase mismatch between the low-level vertical velocity (say $w_{l l}$ ) and the diabatic heating rate $(h)$ for "shallow" $(\tilde{\lambda}<1)$ systems, and strong amplitude sensitivity of the response for "deeper" $(\tilde{\lambda}>1)$ systems. The subsequent discussion hinged on the premise that a ' $w_{l l}-h$ ' phase match is desirable on physical grounds, and the attendant postulate that such a relationship should be sought for, or enforced, in an NWP model assimilation scheme for condensational heating. In effect a form of inverse problem was posed and a search was made for other vertical diabatic profiles that would ensure satisfaction of this desired criterion. It was shown that the inclusion of evaporative cooling in a sub-cloud layer can possibly alleviate the problem linked with shallow systems, and that an asymmetry in the relative disposition of the diabatic effects between the upper and lower regions of the cloud provides a possible mechanism to counter/cure phase mis-matching for deep systems. In contrast for diabatic distributions characterised by $R_{0}<1$, the evanescent vertical structure associated with such flows ensure vertical coherency and phase matching of the response. However, there also remains in this case a not insignificant amplitude sensitivity of the response to the precise shape of the vertical diabatic profile.

This examination of the inverse problem is clearly not exhaustive and hence this, along with the idealised nature of the model, imposes limitations upon the general applicability of our inferences. Major restrictions are associated with the special nature of the diabatic profiles considered here, and the omission from the model of some of the key 
features of mesoscale weather systems. For example the condensational heating and cooling implied by (2.2) in conjunction with (2.5), (and even evaporative heating and cooling in conjunction with 2.10) is a not unreasonable theoretical contrivance for studying the horizontally non-dispersive meso- $\beta$ scale regime and also for the $R_{0}<1$ regime, but it may restrict the results to be of little more than qualitative interest elsewhere. Again it could be argued that the amplitude sensitivity recorded in sections 2.2 and 2.3 is a direct consequence of the enforced stipulation of steady (as opposed to growing or decaying) condensational activity. There are two suggestive partial counters of this argument. Many long-lived mesoscale systems do possess a comparatively prolonged quasi-steady phase. Also the results of theoretical wave-CISK studies indicate that, for a given atmospheric structure, the phase behaviour of CISK modes of moderate growth is akin to, at least to the first order, the properties of the neutral mode associated with that same mean atmospheric structure. The limitations imposed by the linearity of the model and the omission of certain key physical mechanisms is probably much more severe. However, it is of interest to record that the addition of a linear vertically sheared transverse flow component $U=\Lambda z$ into the basic flow field of the model (i.e. modification of the base system of quations 2.1), in accompaniment with a profile (b) distribution for the diabatic heating, appears to only qualitatively modify the response given by (2.6c).

The foregoing comments serve to underline the rudimentary and exploratory nature of the present study. Thus it follow that our inferences regarding the flow response to the assimilation of cloud condensational effects constitute mere cautionary and tentative remarks. However on the basis of these inferences it would at least be of some interest to examine the sensitivity of NWP models to the specification of the vertical profile of the diabatic heating during an assimilation (or initialization) operation. A series of experiments conducted with different vertical diabatic heating profiles and ac- tive "cloud" regions of differing horizontal extent would provide base information. An assessment of the sensitivity could be obtained in the usual way from an examination of the structure and amplitude of the "difference fields" derived from the results of the various simulations. Such an excercise could be designed in terms of, and would test, the inferences drawn in the present study.

\section{References}

Austin, G.L., 1985: Application of pattern recognition and extrapolation techniques to forecasting. ESA Journal, 85/2, 147-156.

Chang, C-P., 1976: Vertical structure of tropical waves maintained by internally-induced cumulus heating. J. Atmos. Sci., 33, 729-739.

Danard, M., 1985: On the use of satellite of precipitation in initial analyses for numerical weather prediction. Atmos.-Ocean, 23, 23-42.

Davies, H.C., 1979: Phase-lagged wave-CISK. Quart. J. Roy. Met. Soc., 105, 325-364.

Davies, H.C., 1980: An ubiquitous property of simple wave-CISK models and its implications. J. Atmos. Sci., 37, 1437-1446.

Fiorino, M. and T. T. Warner, 1981: Incorporating surface winds and rainfall rates into the initialization of a mesoscale hurricane model. Mon. Wea. Rev., 109, 1914-1929.

Hayashi, Y., 1976: Non-singular resonance of equatorial waves under the radiation condition. J. Atmos. Sci., 33, 183-201.

Molinari, J., 1982: Numerical hurricane prediction using assimilation of remotely sensed rainfall rates. Mon. Wea. Rev., 110, 553-571.

Ninomiya, K., 1987: Mesoscale numerical weather prediction-Numericale prediction of mesoscale severe phenomena in Japan-, Short- and MediumRange Numerical Weather Prediction (This volume), 517-531.

Raymond, D. J., 1986: Prescribed heating of a stratified atmosphere as a model for moist convection. J. Atmos. Sci., 43, 1101-1111.

Roesli, H.P., 1981: The Swiss network of two digital radars and 60 automatic weather stations. Proc. Nowcasting-I, Hamburg, Germany, ESA SP. 165, 317-322.

Smith, R.B. and Y.L. Lin., 1982: The addition of heat to a stratified air-stream with application to the dynamics of orographic rain. Quart. J.R. Met. Soc., 108, 353-378.

Tarbell, T.C., T.T. Warner, and R.A. Anthes, 1981: An example of the initialization of the divergent wind component in a mesoscale numerical weather prediction model. Mon. Wea. Rev., 109, 77-95.

Tatehira, R., Hitsuma, M. and Y. Makino, 1981: 
The mesoscale observational network in Japan. Proc. Nowcasting-I, Hamburg, Germany, ESA SP-165, 341-346.

Tsonis, A.A. and G.A. Isaac, 1985: On a new approach for instantaneous rain area delineation in the midlatitudes using GOES data. J. Climate Appl. Meteor., 24, 1208-1218.

Ueno, M., Taira, R. and T. Kudoh, 1987: A dyna- mic assimilation method for a mesoscale model using observed rainfall rates. Short-and Medium. Range Numerical Weather Prediction (This volume), 573-584.

Zawadzki, I., 1984: Factors affecting the precision of radar measurements of rain. Proc. 22 AMS Conf. Radar Meteorology, Zurich, 251256. 